\title{
Intraoperative Ketamine and Magnesium Therapy to Control Postoperative Pain After Abdominoplasty and/or Liposuction: A Clinical Randomized Trial
}

This article was published in the following Dove Press journal:

Journal of Pain Research

\author{
Verónica Varas $\mathbb{I D}^{1}$ \\ Paz Bertinelli ${ }^{\prime}$ \\ Pablo Carrasco' \\ Nicole Souper' \\ Patricio Álvarez' \\ Stefan Danilla ${ }^{2}$ \\ José Ignacio Egaña ${ }^{1,3}$ \\ Antonello Penna (iD) ${ }^{1,4}$ \\ Sergio Sepúlveda ${ }^{2}$ \\ Virginia Arancibia' \\ María Gabriela Álvarez' \\ Rodrigo Vergara (iD ${ }^{5}$ \\ 'Department of Anesthesia and \\ Perioperative Medicine, Hospital Clínico \\ de la Universidad de Chile, Santiago, \\ Chile; ${ }^{2}$ Department of Surgery, Hospital \\ Clínico de la Universidad de Chile, \\ Santiago, Chile; ${ }^{3}$ Biomedical \\ Neuroscience Institute (BNI), \\ Universidad de Chile, Santiago, Chile; \\ ${ }^{4}$ Centro de Investigación Clínica \\ Avanzada (CICA), Hospital Clínico de la \\ Universidad de Chile, Santiago, Chile; \\ ${ }^{5}$ Departamento de Kinesiología, Facultad \\ de Artes y Educación Física, Universidad \\ Metropolitana de Ciencias de la \\ Educación, Santiago, Chile
}

Correspondence: Verónica Varas Departamento de Anestesiología y Medicina Perioperatoria, Hospital Clínico, Universidad de Chile, Santos Dumont 999, Santiago 838 0456, Chile Tel +5622978822l

Email vvaras@uchile.cl
Purpose: This study aimed to compare the effects of ketamine and ketamine associated with magnesium on opioid consumption and pain scores in patients undergoing abdominoplasty and/or liposuction compared to standard treatment.

Patients and Methods: A total of 63 patients were included and randomized as follows: 21 patients in the Control group, 20 patients in the Ketamine group (Ket), and 22 patients in the Ketamine-magnesium group (KetMag). The KetMag group received an IV bolus of $0.3 \mathrm{mg} /$ $\mathrm{kg}$ of ketamine and $50 \mathrm{mg} / \mathrm{kg}$ magnesium, followed by continuous infusion of ketamine $(0.15 \mathrm{mg} / \mathrm{kg} / \mathrm{h})$ and magnesium $(10 \mathrm{mg} / \mathrm{kg} / \mathrm{h})$ until extubation. The Ket group received the same bolus and infusion of ketamine, together with a bolus and continuous infusion of placebo instead of magnesium. The Control group received saline instead of ketamine and magnesium. The groups were compared in morphine consumption during the first $12 \mathrm{~h}$, bodypostoperative pain and disability scale until the 90th day, the time until the first morphine request on the PCA pump, pain scores, and the adverse effects related to the use of study drugs.

Results: The KetMag group had a lower morphine consumption by almost $50 \%$ during the first $12 \mathrm{~h}$ than the Control and the Ket groups. In addition, the KetMag group required the first dose of morphine later than the other two groups. There were no differences in the adverse effects of the proposed treatments. Finally, multiple linear regression and a nonlinear approach analysis indicated that the Control group experienced a higher degree of pain and increased morphine consumption per hour than Ket and KetMag groups.

Conclusion: Co-administration of intraoperative ketamine plus magnesium and ketamine alone are an effective and easy regime for reducing pain and opioid consumption in the postoperative period.

Keywords: abdominoplasty, ketamine, lipectomy, magnesium, analgesics, postoperative pain

\section{Introduction}

Postoperative pain after abdominoplasty and liposuction surgery is described as moderate to severe, frequently requiring large amounts of opioids, which may produce adverse effects such as respiratory depression, sedation, nausea and vomiting, and potentially increasing the risk of addiction. ${ }^{1}$ Thus, analgesia modalities that spare opioid consumption could be beneficial for these patients. 
Ketamine and magnesium sulfate (magnesium from now on) are known N-methyl-d-aspartate (NMDA) antagonists and effective analgesics for postoperative pain, independent of opioid receptors. ${ }^{2-7}$ After major abdominal and orthopedic surgeries, a low dose of ketamine decreases opioid consumption. ${ }^{2,3,8-14}$ Moreover, perioperative infusion of magnesium also diminishes analgesic drug consumption. ${ }^{15-21}$ Finally, one study demonstrated that ketamine plus magnesium further reduces opioid requirements after major orthopedic surgery. ${ }^{8}$ Nevertheless, it is currently unknown whether ketamine and magnesium are useful for treating postoperative pain after abdominoplasty and/or liposuction surgery.

The purpose of this trial is to evaluate intraoperative ketamine and magnesium's efficacy to decrease postoperative opioid consumption for abdominoplasty and liposuction surgery. We hypothesize that ketamine alone reduces postoperative opioid consumption and that its combination with magnesium has even more significant opioid-sparing effects in this type of surgery.

\section{Materials and Methods}

This prospective, randomized, double-blind controlled trial was conducted at Hospital Clínico de la Universidad de Chile. The study was registered at www.clinicaltrials.gov (NCT02450214). The protocol was approved by the local Ethics Committee (Record Number 004-2015 Comité Ético-Científico at Hospital Clínico de la Universidad de Chile). The study was carried out between May 2015 and April 2017. After the patient signed the informed consent, patients undergoing abdominoplasty and/or liposuction surgery were enrolled. The study was conducted under Good Clinical Practice and the Guidelines of the Declaration of Helsinki.

\section{Participants}

Inclusion criteria were age $\geq 18$, American Society of Anesthesiologists (ASA) physical status I-II, with a body mass index $21-28 \mathrm{~kg} / \mathrm{m}^{2}$, and complete secondary education. Exclusion criteria were an inability to consent to the study, the surgery being more complex than initially planned (eg lower body lift with Fleur de Lis abdominoplasty), the use of analgesic drugs consumption $48 \mathrm{~h}$ before surgery, peripheral and central neurologic diseases, prior liposuction, and known allergies to any of the other drug used in the protocol.

\section{Procedure}

All patients were randomly assigned to one of the three following intraoperative treatments: a) Control group
(Control): $50 \mathrm{~mL}$ syringes and $100 \mathrm{~mL}$ bags containing $0.9 \%$ saline solution, b) Ketamine group (Ket): $50 \mathrm{~mL}$ syringes containing $1 \mathrm{mg} / \mathrm{mL}$ ketamine in $0.9 \%$ saline solution and $100 \mathrm{~mL}$ bags of normal saline, and c) Ketaminemagnesium group (KetMag): $50 \mathrm{~mL}$ syringes containing $1 \mathrm{mg} / \mathrm{mL}$ ketamine in $0.9 \%$ saline solution and $100 \mathrm{~mL}$ bags with $50 \mathrm{mg} / \mathrm{mL}$ magnesium in normal saline $0.9 \%$. At the beginning patients received ketamine or normal saline $0.3 \mathrm{~mL} / \mathrm{kg}(0.3 \mathrm{mg} / \mathrm{kg})$ and it was followed by an infusion of $0.15 \mathrm{~mL} / \mathrm{kg} / \mathrm{h}(0.15 \mathrm{mg} / \mathrm{kg} / \mathrm{h})$, while magnesium or normal saline started with a $1 \mathrm{~mL} / \mathrm{kg}(50 \mathrm{mg} / \mathrm{kg})$ bolus (administered in 30 minutes) and then it was infused at $10 \mathrm{mg} / \mathrm{kg} / \mathrm{h}$. We generated the randomization through the free online application Sealed EnvelopeTM (http://www. sealedenvelope.com) in swapped blocks of $\mathrm{n}=3,6$, and 9 , which were stratified by each surgeon participating in the protocol. The code was written and kept in sealed opaque envelopes sequentially. A nurse unrelated to patient management opened the envelope and prepared the code's solution. The anesthesiologist received the drugs (Ketamine, Magnesium, or placebo) with the label "ketamine" and "magnesium sulfate." The anesthesiologist, surgeon, pain evaluator, and patient did not know their allocated solutions protecting the double-blind during the entire process.

All patients received a standardized general anesthesia protocol. Anesthesia induction was performed with $2 \mathrm{mg} / \mathrm{kg}$ of propofol, remifentanil effect-site targeted concentration between 3-5 ng/mL (Minto model), and rocuronium $(0.6 \mathrm{mg} / \mathrm{kg})$. Anesthesia was maintained with $0.8-1.0 \mathrm{mini}-$ mal alveolar concentration (MAC) of sevoflurane, effectsite remifentanil between $3-5 \mathrm{ng} / \mathrm{mL}$, and subsequent doses of rocuronium if needed. The neuromuscular blocking effect was evaluated before extubation using the TOF monitor. If $\mathrm{T} 4 / \mathrm{T} 1$ was $<90 \%$, residual neuromuscular blockade was reversed with $20-60 \mu \mathrm{g} / \mathrm{kg}$ of neostigmine and 10-30 $\mu \mathrm{g} / \mathrm{kg}$ atropine. Antibiotic prophylaxis was carried out with $2 \mathrm{~g}$ of cefazolin (or Clindamycin 600mg in the case of allergy to penicillin). All patients received noninvasive standard monitoring. Before skin incision, the surgeon performed a subcutaneous administration with a 1:1.000.000 adrenaline solution. Thirty minutes before the end of the surgery, all patients received $1 \mathrm{~g}$ of paracetamol with $60 \mathrm{mg}$ of ketorolac in addition to $0.1 \mathrm{mg} / \mathrm{kg}$ of morphine thirty minutes before the end of the surgery. Nausea and vomiting prophylaxis was carried out with $8 \mathrm{mg}$ of dexamethasone after induction and $4 \mathrm{mg}$ of ondansetron. In the postanesthesia care unit (PACU), nausea was treated with $0.625 \mathrm{mg}$ of droperidol. After the surgery, patients spent at 
least two hours in the PACU. The pain and morphine consumption were evaluated by an investigator who was unaware of the treatment group assignments.

Postoperative analgesia included a $90 \mathrm{mg}$ ketorolac in $250 \mathrm{~mL}$ of $0.9 \%$ saline solution infusion every $24 \mathrm{~h}$. A morphine PCA pump was installed and programmed with no continuous infusion, $1 \mathrm{mg}$ boluses, and $8 \mathrm{~min}$ lockout. Thromboembolic prophylaxis was initiated with $10 \mathrm{mg}$ of rivaroxaban (Xarelto ${ }^{\circledR}$, Bayer) per day for ten days; $20 \mathrm{mg}$ of esomeprazole (Nexium ${ }^{\circledR}$, Astra-Zeneca) was administered every $12 \mathrm{~h}$ for seven days as prophylaxis for pharmacological gastritis, and antibiotic prophylaxis with $500 \mathrm{mg}$ of cefadroxil (Cefamox ${ }^{\circledR}$, Merck-Serono) begun every $12 \mathrm{~h}$ for seven days.

\section{Measurements}

Initially, the study's primary outcome was the body-PPDS scale score (postoperative pain and disability scale) on the 90th postoperative day. ${ }^{22}$ The body-PPDS scale score is a patient-reported outcome instrument to measure postoperative pain and discomfort experienced by the patient after surgery, a subjective assessment of general symptoms, inflammatory discomfort, somatic pain, neuropathic pain, mobility, daily activities, wound problems, and body shaping postoperative garment problems. Unfortunately, after recruiting 25 patients, we noted several technical limitations for obtaining the scale score, and it was abandoned. We replaced our primary outcome to morphine consumption (cumulative dose of morphine from PCA pump) in the first $12 \mathrm{~h}$ beginning right after the patient's arrival to the post-anesthesia care unit (PACU).

As secondary outcomes, we included total morphine consumption at 0,2 , and $6 \mathrm{~h}$ after arriving at the PACU, the time until the first morphine request on the PCA pump, and pain scores. The pain was assessed at rest and during movement (trying to change position) recorded at $0,2,6,12$, and $24 \mathrm{~h}$, quantified using an eleven-point numeric rating scale from 0 to 10 where zero represents no pain, and ten the worst pain imaginable. When patients were discharged before $24 \mathrm{~h}$, the pain was assessed by a telephone interview. Demographic data, surgical variables such as the amount of fat aspirated, weight of the resected flap, surgery duration, immediate postoperative complications such as bleeding, hematoma, or thromboembolic were recorded. Finally, we recorded the adverse effects related to the use of study drugs for up to $24 \mathrm{~h}$ after surgery: hallucinations, unpleasant dreams, degree of sedation according to the Ramsay scale, itching, hypotension, bradycardia, urine retention, nausea, and vomiting.

\section{Sample Size}

The sample size was recalculated for the new primary outcome of morphine consumption at $12 \mathrm{~h}$ after surgery, with a type I error of 0.05 and a power of $80 \%$. A sample of 63 patients was required to detect a reduction by $30 \%$ in the morphine consumption at $12 \mathrm{~h}$. G*Power Software, version 3.1 .7

\section{Data Analysis}

We performed two approaches to analyze our data. First, primary and secondary outcomes were analyzed using one-way ANOVA, Kruskal-Wallis test, and Fisher's exact test, as appropriate. Second, because our main objective was to evaluate distinctive dynamics in pain through 12 postoperative hours, we decided to perform more complex analyses to detect differences between groups, which are not evident with the first analysis. Thus, a general approach using multiple linear regression to evaluate how pain score, cumulative morphine consumption, and estimation of by hour morphine consumption changes during these 12 hours was used. Since this dynamic was not linear, we decided to complement this approach with a nonlinear one: regression tree.

For linear regression models, we evaluated the normality of the dependent variable and residuals using the Shapiro-Wilk normality test and heteroscedasticity using the Non-constant Error Variance Test. In general, linear models are robust to assumption violations. $^{23,24}$ Nonetheless, we included the assumption reports in the report of the model. Three multiple linear models were done, one for pain score, another for cumulative morphine consumption, and the third for the morphine per hour consumption ratio as dependent variables. For the morphine per hour consumption, we divided the consumed dose of morphine since the previous evaluation by the time elapsed since then. As such, for the $12 \mathrm{~h}$ measurement, we counted the number of doses administered since hour six, and then we divided that number by six. This ratio allows us to assess how consumption varies along the postoperative period. Independent variables for all the models were the same, Time and Group. Time was entered as continuous variables in hours $(0.33,2,6$, and $12 \mathrm{~h}$ post-surgery), while Group was a dummy variable for Control, Ket, and KetMag groups. We used the Ket group as a reference for the dummy variable. Finally, to evaluate if the dynamic over time was different for each group, we assessed the interaction of Group and Time variables. 
Table I Basal and Intraoperative Characteristics Between Groups

\begin{tabular}{|c|c|c|c|c|}
\hline Characteristics & Control $(n=21)$ & Ketamine $(n=20)$ & KetMag $(n=22)$ & $P$ value \\
\hline Age in years (mean $\pm S D)$ & $40.2(8.5)$ & $39.8(6.1)$ & $39.2(7.2)$ & 0.91 \\
\hline Gender, female (\%) & 100 & 95 & 86 & 0.18 \\
\hline $\mathrm{BMI}$ in kg/m (mean $\pm \mathrm{SD})$ & $25.0(2.0)$ & $25.1(2.2)$ & $25.0(1.5)$ & 0.99 \\
\hline Anesthesia duration in min (median, range) & $300(170-420)$ & $300(230-480)$ & $280(182-405)$ & 0.81 \\
\hline Surgery duration in min (median, range) & $215(160-360)$ & $240(160-300)$ & $220(150-310)$ & 0.59 \\
\hline Remifentanil amount in mg (mean) & 2.58 & 2.40 & 2.51 & 0.51 \\
\hline
\end{tabular}

Abbreviations: KetMag, ketamine and magnesium; SD, standard deviation.

To assess the nonlinear aspect of postoperative dynamic's, we used a conditional regression tree, using morphine consumption as the dependent variable and Group and postoperative time as regressors. We used this procedure to facilitate the detection of specific postoperative periods in which the groups would differ. The pruning procedure was done as suggested by Kabacoff. ${ }^{25}$ All demographic analyses were done using GraphPad Prism 8.0. Linear regression models and conditional regression trees were implemented using R-project. For all statistical procedures performed, an $\alpha=0.05$ was used.

\section{Results}

Sixty-three patients were included and randomized as follows: 21 patients in the Control group, 20 patients in the Ket group, and 22 patients in the KetMag group. All patients were included in the analysis, and groups were comparable in age, gender, ASA physical status, body mass index, surgical and anesthesia duration, and amount of Remifentanil administered (Table 1).

Using the first analytical approach (ANOVA), we observed that patients of the KetMag group had almost
$50 \%$ less morphine-consumption at $12 \mathrm{~h}$ after surgery in comparison with Control and the Ket groups (Figure 1A). The cumulative morphine-consumption during the first $12 \mathrm{~h}$ after surgery also showed that the KetMag group consumed significantly less morphine at $6 \mathrm{~h}$ and $12 \mathrm{~h}$ (Figure 1B). In addition, patients from the KetMag group significantly delayed the administration of the first dose of morphine with regards to the Control group (Figure 2). There were no differences between postoperative nausea and vomiting incidences between groups (Table 2); likewise, no differences were found in sedation or hallucinations. Finally, rest and dynamic pain scores between the three groups were no different at 2, 6 and 12h (Figure 3A and B). However, immediately after arrival to PACU, patients from the Control group demonstrated higher rest and dynamic pain scores than the other 2 groups (Figure $3 \mathrm{~A}$ and $\mathrm{B}$ ).

To perform a more complete and in-depth analysis of pain and morphine consumption, we decided to do a multiple linear regression and a nonlinear approach (regression trees) to evaluate distinctive dynamics in pain and morphine consumption throughout the 12 hours following the operation. First, we analyzed the dynamic of postoperative pain scores
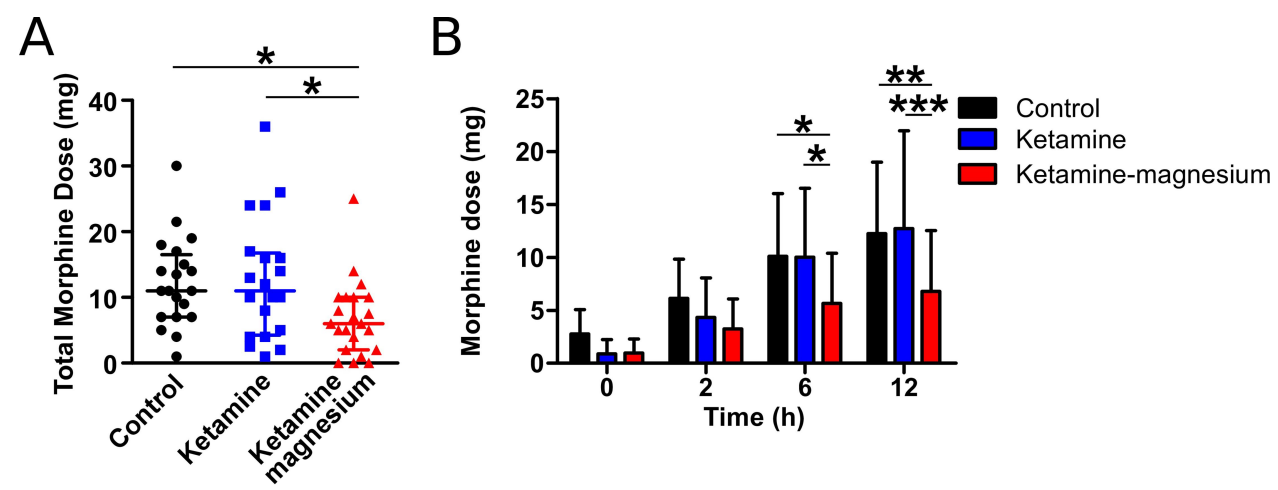

Figure I The morphine consumption by patients using PCA during the first I $2 \mathrm{~h}$ after surgery in all groups. (A) In the cumulative dose of morphine at I2h is observed that Ketamine-magnesium $(n=22)$ consumes significantly less morphine than control $(n=2 I)$ and ketamine $(n=20)$ groups (Kruskal-Wallis test and Dunn's Multiple Comparison test: control vs ketamine-magnesium $\mathrm{p}<0.05$ and ketamine vs ketamine-magnesium $\mathrm{p}<0.05$ ). (B) Cumulative doses of morphine at 0 (PACU), 2 , 6 , and I2h show that ketamine-magnesium groups consume significantly less morphine at 6 and $12 \mathrm{~h}$ (Two-way ANOVA and Bonferroni post hoc test, control vs ketamine-magnesium at 6 and $12 \mathrm{~h}$, $\mathrm{p}<0.05$ and $\mathrm{p}<0.01$; ketamine vs ketamine-magnesium 6 and $12 \mathrm{~h}, \mathrm{p}<0.05$ and $\mathrm{p}<0.00 \mathrm{I})$. ${ }^{*} \mathrm{p}<0.05,{ }^{* *} \mathrm{p}<0.01$, and $* * * \mathrm{p}<0.00 \mathrm{I}$. 


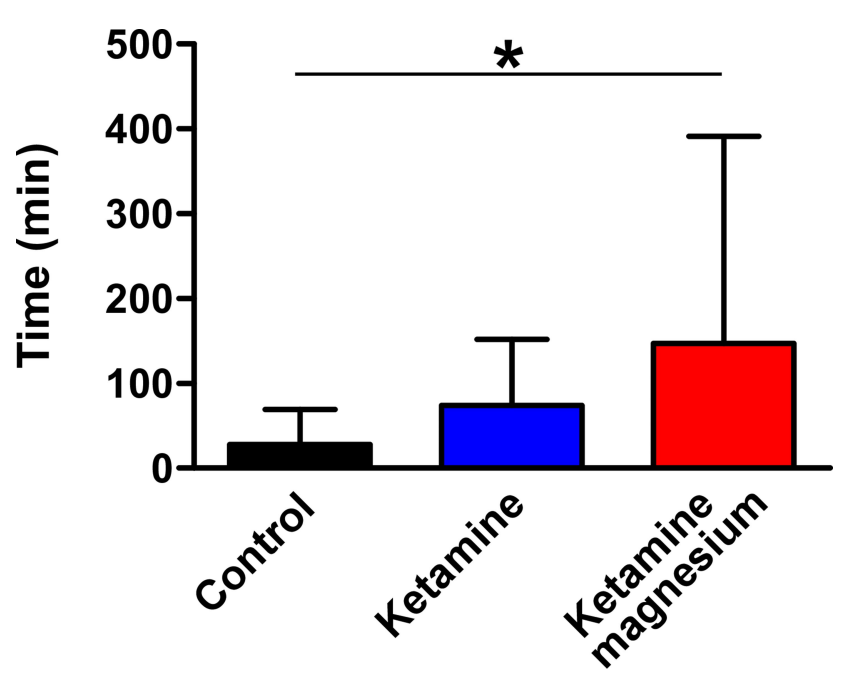

Figure 2 Time to the first morphine requirement of patients using PCA. The graph shows that patients of Ketamine-magnesium significantly delay more the first morphine dose than Control (ANOVA and Dunnett's Multiple Comparison test: control vs ketamine-magnesium $\mathrm{p}<0.05)$. ${ }^{*} \mathrm{p}<0.05$.

and morphine consumption (ratio and cumulative) using multiple linear regressions. In the pain scores (Figure 4A), we found a significant model $(\mathrm{F}(2,242)=5.8, \mathrm{p}=4.3 \mathrm{e}-05$, adjusted $R^{2}=0.08$; Dependent variable Normality: $\mathrm{W}=0.90, \mathrm{p}=2.39 \mathrm{e}-$ 13; Residuals Normality: $\mathrm{W}=0.97, \mathrm{p}=0.0001 ; \chi^{2}=6.72, d f=1$, $\mathrm{p}=0.009$ ), where only the Control and the Ket groups were different (the Ket group as reference: $\beta_{\text {KetMag }}=0.41, \mathrm{t}=0.73$, $\left.\mathrm{p}=0.46, \beta_{\mathrm{Ctrl}}=2.00, \mathrm{t}=3.42, \mathrm{p}=0.0007\right)$, while time were not $\left(\beta_{\text {Time }}=-0.05, t=-0.84, p=0.39\right)$. Nonetheless, we also found that in the time, there was a significant difference between the Control and the Ket groups $(\beta=-0.17, \mathrm{t}=-2.0, \mathrm{p}=0.04)$. This means that the Control group dynamic is different from the Ket and the KetMag groups.

Ratio Morphine Consumption presents a similar pattern to that of Pain score (Figure 4B). The Control group seems to start with higher morphine doses with a marked drop throughout the hours, while the Ket and the KetMag groups start with lower morphine consumption slowly dropping throughout time. These observations are supported by the linear regression analysis which presents a significant model $(\mathrm{F}(5,242)=15.74, \mathrm{p}=2.06 \mathrm{e}-13$, adjusted $R^{2}=0.22$; Dependent variable Normality: $\mathrm{W}=0.84$, p6.31e-

Table 2 Incidence of Nausea and Vomiting Between Groups

\begin{tabular}{|l|l|l|l|l|}
\hline Characteristics & $\begin{array}{l}\text { Control } \\
(\mathbf{n = 2 1})\end{array}$ & $\begin{array}{l}\text { Ketamine } \\
(\mathbf{n}=\mathbf{2 0})\end{array}$ & $\begin{array}{l}\text { KetMag } \\
(\mathbf{n = 2 2})\end{array}$ & P value \\
\hline Nausea (\%) & 42.9 & 35 & 22.7 & 0.37 \\
Vomiting (\%) & 19 & 5 & 13.6 & 0.4 \\
\hline
\end{tabular}

Abbreviation: KetMag, ketamine and magnesium.
15; Residuals Normality: $\mathrm{W}=0.93, \mathrm{p}=4.39 \mathrm{e}-09 ; \chi^{2}=95.6$, $d f=1, \mathrm{p}=2.22 \mathrm{e}-16$ ). In this model, the Ket group was only significantly different from the Control group $(\beta=2.9$, $\mathrm{t}=4.1, \mathrm{p}=3.91 \mathrm{e}-05)$, but not with the KetMag group $(\beta=$ $-0.42, \mathrm{t}=-0.61, \mathrm{p}=0.53)$. Time was a significant predictor of Ratio Morphine Consumption $(\beta=-0.19, \mathrm{t}=-2.6$, $\mathrm{p}=0.008$ ), and in the time the Control group was different than the Ket group $(\beta=-0.31, \mathrm{t}=-3.0, \mathrm{p}=0.002)$, while the KetMag and the Ket groups were not different in the time $(\beta=0.003, \mathrm{t}=0.03, \mathrm{p}=0.97)$.

Cumulative Morphine Consumption (Figure 4C) presents a significant multiple linear model $(F(5,242)=29.4$, $\mathrm{p}=2.2 \mathrm{e}-16, \quad$ adjusted $R^{2}=0.36 ; \quad$ Dependent variable Normality: $\mathrm{W}=0.60, \mathrm{p}=2.22 \mathrm{e}-16$; Residuals Normality: $\left.\mathrm{W}=0.83, \mathrm{p}=1.28 \mathrm{e}-15 ; \chi^{2}=187.8, d f=1, \mathrm{p}=2.22 \mathrm{e}-16\right)$. In this model, only Time presented a significant effect $(\beta=0.90$, $\mathrm{t}=7.26, \mathrm{p}=5.01 \mathrm{e}-12$ ). However, we also found a significant difference in the time between the Ket and the KetMag groups $(\beta=-0.46, t=-2.70, p=0.007)$, but not between Ket and Control group. Overall, these results support that Cumulative Morphine Consumption presents a lower slope in the Control and the Ket groups than the KetMag group.

Using regression trees analysis, we first determined if the differences in Ratio Morphine Consumption were secondary to the group and/or postoperative time. As presented in the tree depicted in Figure 5, after arrival to PACU (20 min or $0.33 \mathrm{~h}$ after surgery), the Control group presented a higher morphine consumption than Ket and the KetMag groups. Over the 20 initial minutes, the tree showed a gradual reduction in Ratio Morphine Consumption, which cannot be attributed to group differences. Furthermore, the age of the patients was a significant predictor of morphine consumption at more than $6 \mathrm{~h}$ after surgery. People older than 40 years tended to present lower morphine consumption during the last postoperative hours followed. Finally, the tree showed that Morphine Consumption was diminished with time.

\section{Discussion}

In this randomized controlled trial, we demonstrated that the administration of intraoperative ketamine plus magnesium reduces morphine consumption by almost $50 \%$ during the first $12 \mathrm{~h}$ in patients subjected to abdominoplasty and liposuction compared to patients controls and those who did not receive magnesium associated with ketamine. Additionally, intraoperative infusion of ketamine plus magnesium delayed the first morphine request, while there were no differences in the proposed treatment's 

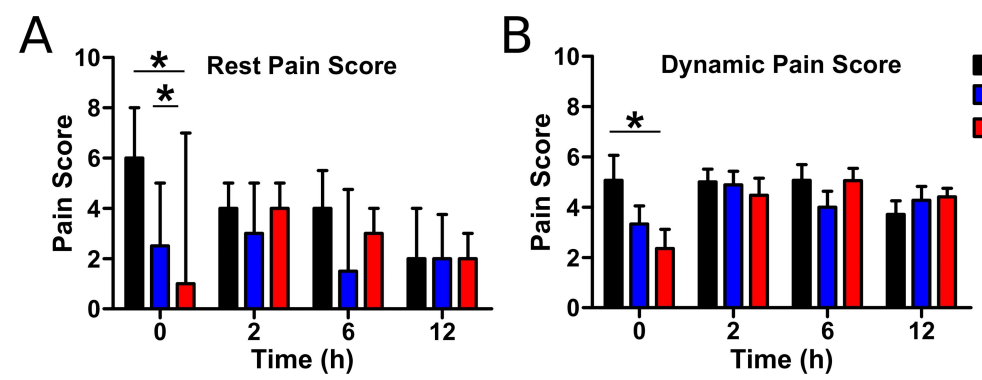

Figure 3 Pain score after surgery until 12 hours in all groups of patients. (A) In the pain score at rest, is observed that the Control group had more pain at Oh (PACU) than the other two groups (Two-way ANOVA and Bonferroni post hoc test, Control vs Ketamine and Control vs Ketamine-magnesium at 0 h, $\mathrm{p}<0.05$ and $\mathrm{p}<0.05$ ). (B) In the dynamic pain score is shown that the Control group had more score of pain at Oh (PACU) than the Ketamine-magnesium group (Two-way ANOVA and Bonferroni post hoc test, Control vs Ketamine-magnesium at 0 h, $\mathrm{p}<0.05)$. ${ }^{*} \mathrm{p}<0.05$.

adverse effects. However, pain scores were similar among all groups. Nevertheless, multiple linear regression and nonlinear approach analyses indicate that the Control group had more pain and consumed more morphine per hour than the ketamine and the ketamine plus magnesium groups. Thus, ketamine and magnesium are effective analgesic drugs with an opioid-sparing effect in patients who undergo abdominoplasty and liposuction.

Recently, the "Consensus Guidelines on the Use of Intravenous Ketamine Infusions for Acute Pain Management" from the American Society of Regional Anesthesia and Pain Medicine, The American Academy of Pain Medicine, and the American Society of Anesthesiologist (2018) conclude that a subanesthetic ketamine dose remains as a powerful and inexpensive tool for practitioners who manage acute pain. ${ }^{26}$ The guidelines recommended that the ketamine bolus dose do not exceed $0.35 \mathrm{mg} / \mathrm{kg}$ and infusions do not exceed $1 \mathrm{mg} / \mathrm{kg} / \mathrm{h}$; however, this higher dose, although it is more effective, has a higher incidence of adverse effects, such as hallucinations, nightmares, nausea, blurred vision, and dizziness. On the other hand, the efficiency of too low doses has inconclusive evidence. In this context, we used low doses of ketamine (bolus $0.3 \mathrm{mg} / \mathrm{kg}$ and infusion $0.15 \mathrm{mg} / \mathrm{kg} / \mathrm{h}$ ), which effectively decrease the morphine consumption in the treatment of acute pain. Sen et al (2009) showed a 35\% reduction in morphine use during the first $24 \mathrm{~h}$ after an abdominal hysterectomy, ${ }^{27}$ with a similar ketamine dose as in our regime. Likewise, Aida et al (2000) found that in patients undergoing distal or total gastrectomy, patients that were administered ketamine had a lower cumulative morphine consumption than patients administered the placebo. Nevertheless, this study used higher doses of ketamine than our study $(1 \mathrm{mg} / \mathrm{kg}$ and then maintained $0.5 \mathrm{mg} / \mathrm{Kg} / \mathrm{h}){ }^{13}$

A previous report by Jabbour et al (2014) demonstrated, in scoliosis surgery, that intraoperative use of ketamine and magnesium reduced postoperative morphine consumption by $30 \%$ compared with ketamine alone and suggested a synergistic effect. ${ }^{8}$ They used the same ketamine low dose as us. In this context, it would seem attractive to use low doses of ketamine, with few adverse effects, and synergize his opioid-sparing effect with another NMDA receptor antagonist. In our study, morphine consumption was reduced by co-administration of intraoperative ketamine plus magnesium and ketamine alone, indicating that magnesium and ketamine could play a critical role in the postoperative opioid-sparing effect.

The effect of ketamine alone was only observed using multiple linear regression and nonlinear approach analysis. In contrast, the co-administration of ketamine and magnesium was assessed using one-way ANOVA. The multiple linear regression and a nonlinear approach analysis are more sensitive to find differences because they independently study each subject's behavior. Then, the subjects are grouped to detect any difference. Thus, both analyses may suggest a clinical synergy between ketamine and magnesium, as both are NMDA receptor antagonists. Indeed, Liu et al already demonstrated that this combination acts in a supra-additive manner. ${ }^{28}$ A further trial comparing a ketamine-magnesium and magnesium only group is needed to test this hypothesis.

The difference in pain score was observed mainly in the first minutes after arrival at PACU. The Control group had more pain immediately after surgery. However, this higher level of pain in the control group was lost at 2, 6, and $12 \mathrm{~h}$. One explanation is that after arrival at PACU, the Control group required more morphine than the other two groups; thus, pain scores in a longer period were comparable. This may represent a surrogate measure of pain relief that the pain score 
A

Ketamine

Control

Ketamine-magnesium

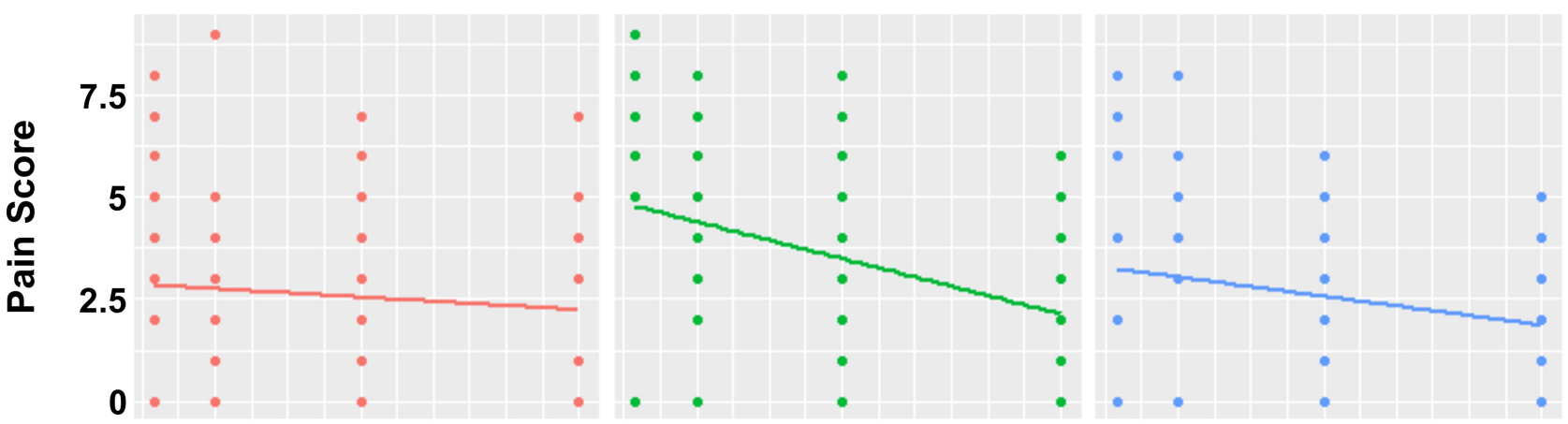

B
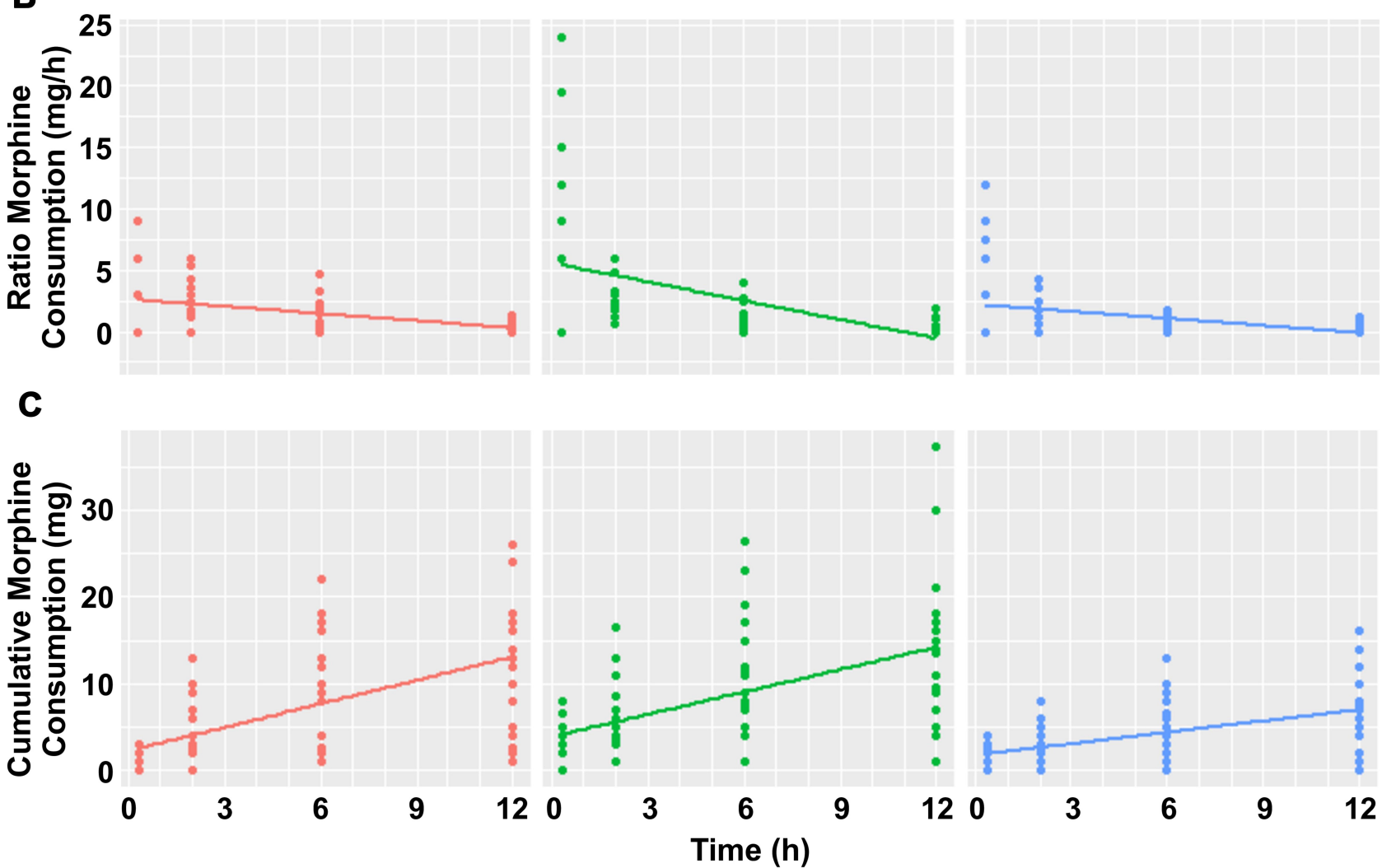

Time (h)

Figure 4 Multiple linear regression models for Pain score, Ratio Morphine Consumption, and Cumulative Morphine Consumptions between all groups. (A) Pain score, the Control group had higher pain from the Ketamine and the Ketamine-magnesium groups $(p<0.001)$, whereas the Control group shows a higher decrease with the time than the other two groups $(p<0.05)$. (B) Ratio Morphine Consumption, the Control group had higher consumption of morphine per hour than Ketamine and Ketaminemagnesium groups $(\mathrm{p}<0.00 \mathrm{I})$, which decay faster in the Control group than the other two groups $(\mathrm{p}<0.0 \mathrm{I})$. (C) Cumulative Morphine Consumption, The Control and the Ketamine groups accumulated more morphine than the Ketamine-magnesium group $(p<0.01)$.

scale was unable to detect. However, despite fewer morphine requirements, postoperative nausea and vomiting incidence was not significantly different among groups. It is likely that we did not find any difference either because all patients received double prophylaxis for PONV, ${ }^{14}$ or the sample size was not enough to see any significant difference. Finally, adverse drug reactions related to ketamine and magnesium were not found; however, the study was not powered to detect the differences of this outcome. Hence, intraoperative ketamine and magnesium therapy are reasonable, effective, and inexpensive to reduce pain and postoperative opioid consumption.

This study was performed in a plastic surgery model; furthermore, the co-administration of ketamine and magnesium might be useful in other clinical scenarios. This strategy would also decrease the risk of opioid abuse in an era of pandemic opioid addiction. Further trials are 


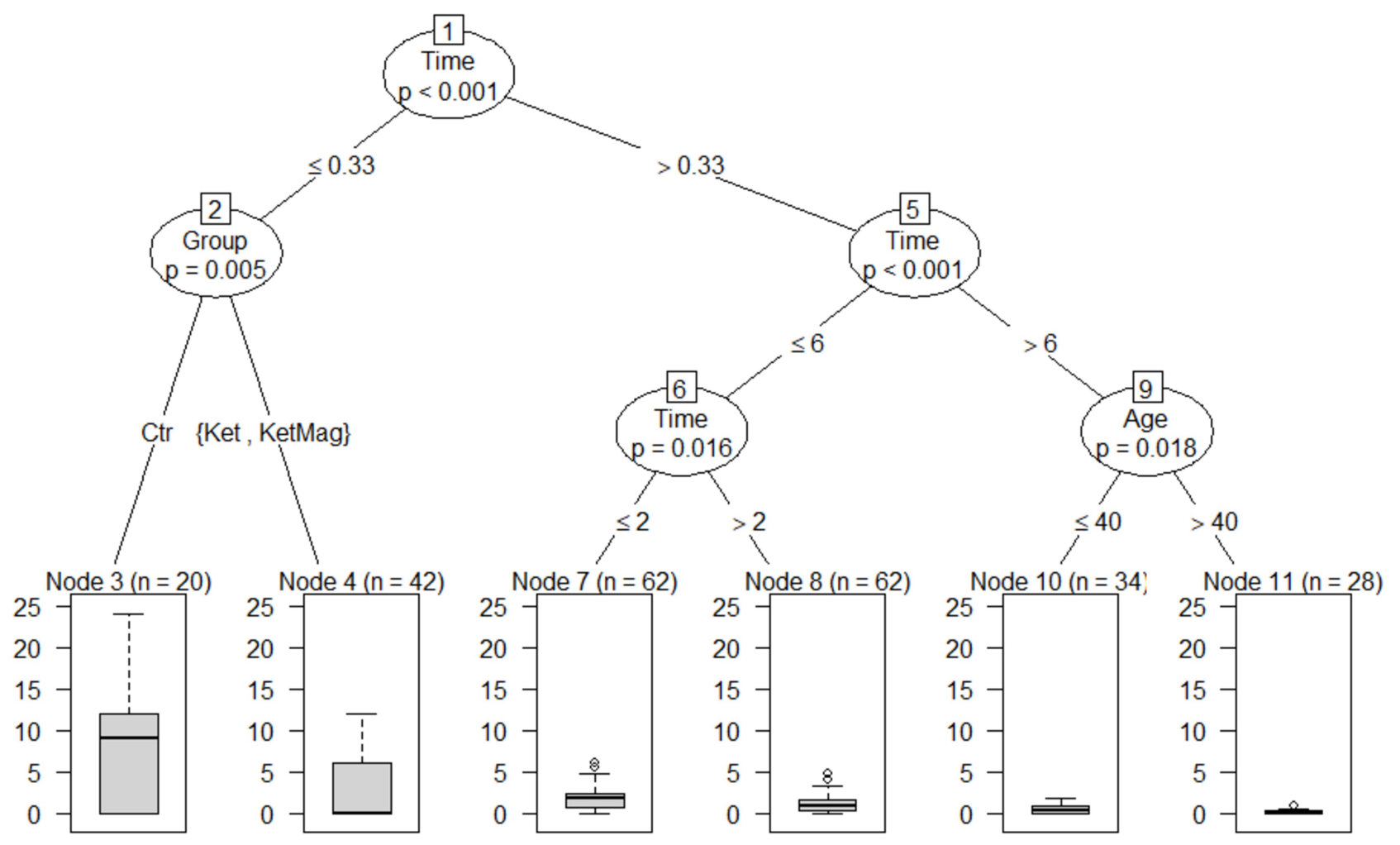

Figure 5 Regression tree analysis for Ratio Morphine Consumption between groups. Node I shows that morphine consumption per hour was higher during PACU (<0.33h) than after PACU stay $(>0.33 \mathrm{~h})(\mathrm{p}<0.00 \mathrm{I})$. In node 2 depicts that at PACU the Control group consumed more morphine per hour than the Ketamine and the Ketaminemagnesium groups $(p=0.005)$. Node 5 and 6 show that morphine's consume per hour decayed after $2 h(p=0.016)$, and after $6 h(p<0.001)$ of surgery. Finally, node 9 shows that patients older than 40 years old consumed less morphine per hour after $6 \mathrm{~h}$ of surgery than younger patients $(p=0.018)$.

required to confirm the results of this study in different clinical scenarios and determine the ideal dosage of these NMDA antagonists in this and other painful surgeries.

A meta-analysis published by McNicol et al (2014) found statistically significant reductions in risk of developing persistent post-surgical pain at 3 and 6 months when intravenous ketamine was used. ${ }^{4}$ However, they reported high variable timing and dosing of ketamine in trials included, suggesting that no unifying effective regimen has emerged. Moreover, based on the variability of the anesthetic and analgesic regimens employed, they suggested increased ketamine efficacy when used as part of a multimodal regime. ${ }^{4}$ Unfortunately, our long-term follow-up was under-reported using the body-PPDS scale score.

The limitations of our study reside in the design and patients included in this study. First, the group size was insufficient to detect a difference in pain scores and the adverse effects of opioids. Second, a group only treated with magnesium was omitted; thus, the magnesium effect was not reported. Third, as plastic surgery is still more frequent in women, in our study, there was an unbalance between sexes, which implies that the conclusions can be applied to women rather than to men. Finally, at the beginning of the study, a long-term follow-up was considered with the body-PPDS scale score, but only $15-21 \%$ of patients answered the questionnaire 90 days after surgery, limiting our original plan to determine the effect of ketamine and magnesium on postoperative chronic pain incidence after plastic surgery.

\section{Conclusion}

In conclusion, this study demonstrates that the coadministration of intraoperative ketamine plus magnesium and ketamine alone are an effective and easy regimen to reduce pain and opioids consumption in the postoperative period.

\section{Data Sharing Statement}

The data sets generated for this study are available on request to the corresponding author (vvaras@uchile.cl). Items to be shared include individual deidentified patient data and studyrelated documents, like study protocol, informed consent, consort chart. These documents will be accessible for ten years from the date of study publication. 


\section{Acknowledgments}

We would like to thank Isabel Zamorano for her assistance in writing the article and to all the clinical teams who participated in this study: nurses, technical staff, and residents that contributed to the realization of this project.

\section{Disclosure}

The authors report no conflicts of interest in this work and this research did not receive any specific grant from funding agencies in the public, commercial, or not-for-profit sectors.

\section{References}

1. Constantine FC, Matarasso A. Putting it all together. Plast Reconstr Surg. 2014;134:113S-119S. doi:10.1097/PRS.0000000000000669

2. Guignard B, Coste C, Costes H, et al. Supplementing desflurane-remifentanil anesthesia with small-dose ketamine reduces perioperative opioid analgesic requirements. Anesth Analg. 2002;95 (1):103-108. doi:10.1097/00000539-200207000-00018

3. Laskowski K, Stirling A, McKay WP, Lim HJ. A systematic review of intravenous ketamine for postoperative analgesia. Can J Anesth. 2011;58(10):911-923. doi:10.1007/s12630-011-9560-0

4. McNicol ED, Schumann R, Haroutounian S. A systematic review and meta-analysis of ketamine for the prevention of persistent post-surgical pain. Acta Anaesthesiol Scand. 2014;58(10):1199-1213. doi:10.1111/ aas. 12377

5. De Oliveira GS, Castro-Alves LJ, Khan JH, McCarthy RJ. Perioperative systemic magnesium to minimize postoperative pain meta-analysis of randomized controlled trials. Anesthesiology. 2013;119(1):178-190. doi:10.1097/ALN.0b013e318297630d

6. Albrecht E, Kirkham KR, Liu SS, Brull R. Peri-operative intravenous administration of magnesium sulphate and postoperative pain: a meta-analysis. Anaesthesia. 2013;68(1):79-90. doi:10.1111/j.13652044.2012.07335.x

7. Murphy JD, Paskaradevan J, Eisler LL, et al. Analgesic efficacy of continuous intravenous magnesium infusion as an adjuvant to morphine for postoperative analgesia: a systematic review and meta-analysis. Middle East J Anaesthesiol. 2013;22(1):11-20.

8. Jabbour HJ, Naccache NM, Jawish RJ, et al. Ketamine and magnesium association reduces morphine consumption after scoliosis surgery: prospective randomised double-blind study. Acta Anaesthesiol Scand. 2014;58(5):572-579. doi:10.1111/aas.12304

9. Assouline B, Tramèr MR, Kreienbühl L, Elia N. Benefit and harm of adding ketamine to an opioid in a patient-controlled analgesia device for the control of postoperative pain: systematic review and meta-analyses of randomized controlled trials with trial sequential analyses. Pain. 2016;157(12):2854-2864. doi:10.1097/j.pain.000000 0000000705

10. Ding X, Jin S, Niu X, et al. Morphine with adjuvant ketamine versus higher dose of morphine alone for acute pain: a meta-analysis. Int J Clin Exp Med. 2014;7(9):2504-2510.

11. Yang L, Zhang J, Zhang Z, Zhang C, Zhao D, Li J. Preemptive analgesia effects of ketamine in patients undergoing surgery. A meta-analysis. Acta Cir Bras. 2014;29(12):819-825. doi:10.1590/ S0102-86502014001900009

12. Jouguelet-Lacoste J, La Colla L, Schilling D, Chelly JE. The use of intravenous infusion or single dose of low-dose ketamine for postoperative analgesia: a review of the current literature. Pain Med. 2015;16(2):383-403. doi:10.1111/pme.12619
13. Aida S, Yamakura T, Baba H, Taga K, Fukuda S, Shimoji K. Preemptive analgesia by intravenous low-dose ketamine and epidural morphine in gastrectomy: a randomized double-blind study. Anesthesiology. 2000;92 (6):1624-1630. doi:10.1097/00000542-200006000-00020

14. Wang L, Johnston B, Kaushal A, Cheng D, Zhu F, Martin J. Ketamine added to morphine or hydromorphone patient-controlled analgesia for acute postoperative pain in adults: a systematic review and meta-analysis of randomized trials.. Can J Anesth. 2016;63 (3):311-325. doi:10.1007/s12630-015-0551-4

15. Levaux C, Bonhomme V, Dewandre PY, Brichant JF, Hans P. Effect of intra-operative magnesium sulphate on pain relief and patient comfort after major lumbar orthopaedic surgery. Anaesthesia. 2003;58(2):131-135. doi:10.1046/j.1365-2044.2003.02999.x

16. Koinig H, Wallner T, Marhofer P, Andel H, Horauf K, Mayer N. Magnesium sulfate reduces intra- and postoperative analgesic requirements. Anesth Analg. 1998;87(1):206-210. doi:10.1213/ 00000539-199807000-00042

17. Bhatia A, Kashyap L, Pawar DK, Trikha A. Effect of intraoperative magnesium infusion on perioperative analgesia in open cholecystectomy. J Clin Anesth. 2004;16(4):262-265. doi:10.1016/j. jclinane.2003.08.012

18. Zarauza R, Sáez-Fernández AN, Iribarren MJ, et al. A comparative study with oral nifedipine, intravenous nimodipine, and magnesium sulfate in postoperative analgesia. Anesth Analg. 2000;91 (4):938-943. doi:10.1097/00000539-200010000-00032

19. Tramèr MR, Schneider J, Marti RA, Rifat K. Role of magnesium sulfate in postoperative analgesia. Anesthesiology. 1996;84 (2):340-347. doi:10.1097/00000542-199602000-00011

20. Steinlechner B, Dworschak M, Birkenberg B, et al. Magnesium moderately decreases remifentanil dosage required for pain management after cardiac surgery. Br J Anaesth. 2006;96(4):444-449. doi:10.1093/bja/ael037

21. Ryu JH, Kang MH, Park KS, Do SH. Effects of magnesium sulphate on intraoperative anaesthetic requirements and postoperative analgesia in gynaecology patients receiving total intravenous anaesthesia. Br J Anaesth. 2008;100(3):397-403. doi:10.1093/bja/aem407

22. Danilla $S$, Cuevas $P$, Aedo $S$, et al. Introducing the body-qol ${ }^{\circledR}$ : a new patient-reported outcome instrument for measuring body satisfactionrelated quality of life in aesthetic and post-bariatric body contouring patients. Aesthetic Plast Surg. 2016;40(1):19-29. doi:10.1007/ s00266-015-0586-5

23. Arnau J, Bendayan R, Blanca MJ, Bono R. The effect of skewness and kurtosis on the robustness of linear mixed models. Behav Res Methods. 2013;45(3):873-879. doi:10.3758/s13428-012-0306-X

24. Schmider E, Ziegler M, Danay E, Beyer L, Bühner M. Is it really robust?: reinvestigating the robustness of ANOVA against violations of the normal distribution assumption. Methodology. 2010;6 (4):147-151. doi:10.1027/1614-2241/a000016

25. Kabacoff R. R in Action: Data Analysis and Graphics with R. $2^{\text {nd }}$ ed. Manning Publications; 2015. Available from: https://www.amazon.co. uk/Action-Data-Analysis-Graphics/dp/1617291382. Accessed August $8,2020$.

26. Schwenk ES, Viscusi ER, Buvanendran A, et al. Consensus guidelines on the use of intravenous ketamine infusions for acute pain management from the American Society of Regional Anesthesia and Pain Medicine, the American Academy of Pain Medicine, and the American Society of Anesthesiologists. Reg Anesth Pain Med. 2018;43(5):456-466. doi:10.1097/AAP.0000000000000806

27. Sen H, Sizlan A, Yanarates O, et al. A comparison of gabapentin and ketamine in acute and chronic pain after hysterectomy. Anesth Analg. 2009;109(5):1645-1650. doi:10.1213/ANE.0b013e3181b65ea0

28. Liu HT, Hollmann MW, Liu WH, Hoenemann CW, Durieux ME. Modulation of NMDA receptor function by ketamine and magnesium: part I. Anesth Analg. 2001;92(5):1173-1181. doi:10.1097/ 00000539-200105000-00019 
Journal of Pain Research

Dovepress

\section{Publish your work in this journal}

The Journal of Pain Research is an international, peer reviewed, open access, online journal that welcomes laboratory and clinical findings in the fields of pain research and the prevention and management of pain. Original research, reviews, symposium reports, hypothesis formation and commentaries are all considered for publication. The manuscript management system is completely online and includes a very quick and fair peer-review system, which is all easy to use. Visit http:// www.dovepress.com/testimonials.php to read real quotes from published authors.

Submit your manuscript here: https://www.dovepress.com/journal-of-pain-research-journal 